\title{
PREVALÊNCIA DE SÍNDROME METABÓLICA E PERFIL NUTRICIONAL DE PACIENTES OBESOS CARDIOPATAS HOSPITALIZADOS
}

\section{METABOLIC SYNDROME PREVALENCE AND NUTRITIONAL STATUS OF OBESE CARDIAC PATIENTS HOSPITALIZED}

\author{
Paula Rodrigues da Costa ${ }^{1}$, Aline Thaís Vieira de Souzaํ, Fabiane La Flor Ziegler \\ Sanches $^{2^{*}}$
}

\begin{abstract}
${ }^{1}$ Egressa do Curso de Nutrição do Faculdade de Ciências Farmacêuticas, Alimentos e Nutrição (Facfan) da Universidade Federal do Mato Grosso do Sul (UFMS). ${ }^{2}$ Docente do Curso de Nutrição da Faculdade de Ciências Farmacêuticas, Alimentos e Nutrição (Facfan); Docente do Programa de PósGraduação em Biotecnologia da Facfan da Universidade Federal do Mato Grosso do Sul (UFMS).

*Autor correspondente: fabiane.sanches@ufms.br, https://orcid.org/0000-0002-0096-3697
\end{abstract}

DOI: $10.35984 / f j h . v 2 i 2.206$

\section{RESUMO}

A Síndrome metabólica é considerada uma doença crônica não transmissível complexa, associada a um conjunto de riscos cardiometabólicos, dentre eles: dislipidemias, intolerância à glicose, resistência à insulina, hipertensão e obesidade. Objetivos: Avaliar a prevalência de Síndrome Metabólica e o perfil nutricional de pacientes obesos cardiopatas de um Hospital Universitário. Estudo transversal com 33 pacientes obesos cardiopatas de um hospital universitário de Campo Grande - MS. Avaliou-se: peso, altura, circunferência da cintura, inquérito de frequência alimentar, pressão arterial, glicose em jejum, níveis séricos de HDL-C e de triglicerídeos. Para prevalência da SM utilizou-se critérios do National Cholesterol Education Program's Adult Treatment Panel III (NCEP-ATP III, 2002). Foi utilizado o software SPSS, considerando nível de significância de 5\%. Resultados: O tipo de cardiopatia e comorbidade mais frequentes foram Infarto Agudo do Miocárdio $(30,3 \%)$ e a hipertensão $(36,6 \%)$, respectivamente. Quanto ao perfil nutricional, clínico e bioquímico, todos os participantes apresentaram elevados níveis de circunferência da cintura, de glicose de jejum e de triglicerídeos, bem como, reduzido nível de HDL-c, porém sem diferença significativa entre os grupos adultos e idosos. Não foram identificadas diferenças significativas no consumo alimentar entre os grupos avaliados. Verificou-se alta prevalência de Síndrome Metabólica nos obesos cardiopatas avaliados (84,85\%), sendo $85,71 \%$ entre os adultos e $100 \%$ entre os idosos. Conclusões: A associação de alterações no perfil nutricional, clínico e bioquímico, que caracterizaram a alta prevalência de síndrome metabólica nos pacientes avaliados, juntamente com os fatores hereditários, reforçam a necessidade de implantação de medidas preventivas para atenuar essa condição.

Palavras-chave: Doenças cardiovasculares; Obesidade; Antropometria; Inquéritos nutricionais; Doença Crônica.

\begin{abstract}
Background: Metabolic syndrome is considered a non-communicable chronic diseases complex, associated with a range of cardiometabolic risk, including: dyslipidemia, glucose intolerance, insulin resistance, hypertension and obesity. Objectives: To evaluate the prevalence of metabolic syndrome and the nutritional status of cardiac obese patients of a university hospital. Methods: Cross-sectional study with 33 obese patients with heart disease of a university hospital in Campo Grande - MS. Were evaluated: weight, height, waist circumference, survey feed rate, blood pressure, fasting glucose, serum levels of HDL-C and triglycerides. For prevalence of MS was used the criteria of the National Cholesterol Education Program's Adult Treatment Panel III (NCEP-ATP III, 2002). We used the SPSS software, considering a significance level of 5\% Results: The type of more frequent heart disease and
\end{abstract}

Recebido: $15 / 05 / 2020$

FAG Journal of Health - ISSN 2674-550X, 2020, v.2, n.2, p. 259

Revisado: 28/05/2020

Aceito: 05/06/2020

Blind reviewers 
comorbidity were Acute Myocardial Infarction (30.3\%) and hypertension (36.6\%), respectively. As for the nutritional, clinical and biochemical profile, all participants had high levels of waist circumference in fasting triglycerides and glucose, as well as reduced levels of HDL-C, but with no significant difference between adults and elderly groups. No significant differences were identified in food consumption among the groups. There was a high prevalence of metabolic syndrome in obese cardiac patients evaluated (84.85\%) and $85.71 \%$ among adults and $100 \%$ among the elderly. Conclusions: The change of association in the nutritional, clinical and biochemical profile which characterized the high prevalence of metabolic syndrome in patients evaluated, along with hereditary factors, reinforce the need to implement preventive measures to alleviate the condition.

Keywords: Cardiovascular diseases; Obesity; Anthropometry; Nutrition surveys; Chronic disease.

\section{INTRODUÇÃO}

As Doenças Crônicas Não Transmissíveis (DCNT), entre elas a obesidade, diabetes, hipertensão e doenças cardiovasculares, representam uma ameaça ao desenvolvimento de todas as nações, pois ocasionam sérios danos e um considerável impacto no sistema de saúde. Em 2008, as DCNT foram responsáveis por 36 milhões de mortes em todo o mundo, sendo que $80 \%$ das mortes ocorreram em países de renda média a baixa (MALTA, SILVA, 2013; WORLD HEALTH ORGANIZATION, 2010). Caracterizada pelo excesso de gordura corporal de um indivíduo, a obesidade é desencadeadora de diversas DCNT, sendo esse um dado crítico tendo em vista o futuro impacto na má qualidade de vida e aumento na morbidade (SHILS et al., 2009).

Conforme a definição da National Cholesterol Education Program's Adult Treatment Panel III, a Síndrome Metabólica (SM) pode ser diagnosticada por critérios, dos quais o indivíduo deve possuir pelo menos três destes componentes: circunferência da cintura elevada ( $>88 \mathrm{~cm}$ para mulheres e $>102 \mathrm{~cm}$ para homens), triglicerídeos séricos elevados ( $\geq 150 \mathrm{mg} / \mathrm{dL}$ ), HDL-colesterol sérico com valores reduzidos ( $<40 \mathrm{mg} / \mathrm{dL}$ para homens e $<50 \mathrm{mg} / \mathrm{dL}$ para mulheres), pressão arterial elevada ( $\geq 130 / 85 \mathrm{mmHg}$ ) e glicemia de jejum elevada $(\geq 110 \mathrm{mg} / \mathrm{dL}$ ), de modo que todas essas variáveis se apresentam associadas ao risco aumentado para diversas cardiopatias (NATIONAL INSTITUTES OF HEALTH, 2001). Sendo também considerada uma DCNT complexa, associada a um conjunto de riscos cardiometabólicos, dentre eles: dislipidemias, intolerância à glicose, resistência à insulina, hipertensão e obesidade (BALLESTRERI, MARCON, TAVARES, 2015).

De acordo com a International Diabetes Federation (IDF) é estimado que aproximadamente 20 a $25 \%$ da população adulta do mundo possuam SM, e que haja duas vezes mais chance de morrer e três vezes mais chance de ter Acidente Vascular Cerebral (AVC) quando comparado com a população que não possui SM (INTERNATIONAL DIABETES FEDERATION, 2014; TIRAPEGUI, 2006).

Uma alimentação inadequada, inatividade física e predisposição genética são fatores que colaboram para o desenvolvimento da SM, sendo a adoção de estilos de vida voltados para a manutenção da saúde por meio de uma alimentação de qualidade e a prática de atividade física elementos básicos para a prevenção da SM (BRASIL, 2005). A nutrição adequada tem como objetivo a redução da circunferência abdominal e gordura visceral, melhora da sensibilidade à insulina e redução da glicemia, prevenindo e retardando o aparecimento de diabetes Tipo 2. De forma geral, recomenda-se a redução no consumo total de gorduras, substituição de gorduras saturadas por insaturadas, diminuição do consumo de gorduras trans, aumento do 
consumo de frutas, hortaliças e cereais integrais, e moderação no consumo de sal e açúcar (BRASIL, 2005).

A necessidade do diagnóstico de SM em ambiente hospitalar faz-se necessária para se ofertar ao paciente um aporte nutricional adequado e consequentemente buscar uma redução no tempo e custos de internação reconhecendo as diversas complicações que estão associadas a essa síndrome. Diante do exposto, o presente trabalho teve como objetivo avaliar a prevalência de SM e o perfil nutricional de pacientes obesos cardiopatas internados no Hospital Universitário Maria Aparecida Pedrossian (NHU) de Campo Grande, Mato Grosso do Sul (MS).

\section{METODOLOGIA}

\subsection{Aspectos éticos, desenho do estudo e população}

Esse projeto foi aprovado pelo Comitê de Ética em Pesquisa da Universidade Federal de Mato Grosso do Sul (UFMS) sob parecer n 981.851, de 11 de março de 2015, em consonância com a Declaração de Helsinki. Os pacientes que concordaram em participar do estudo assinaram o Termo de Consentimento Livre e Esclarecido, considerando-se as recomendações e orientações da resolução no 466, de 12 de dezembro de 2012, do Conselho Nacional de Saúde que regulamenta o desenvolvimento de pesquisas envolvendo seres humanos.

Estudo transversal realizado com pacientes internados na Unidade Coronariana e na enfermaria de Clínica Médica do Hospital Universitário Maria Aparecida Pedrossian (HUMAP) de Campo Grande (MS), no período de abril a agosto de 2015. Foram considerados critérios de inclusão: indivíduos obesos com índice de massa corporal (IMC) $\geq 30 \mathrm{~kg} / \mathrm{m}^{2}$ para adultos e $>27 \mathrm{~kg} / \mathrm{m}^{2}$ para idosos; cardiopatas (angina, estenose aórtica, arritmias, cardiomiopatia, doenças das artérias coronárias, endocardite, infarto do miocárdio e insuficiência cardíaca); com idade igual ou superior a 18 anos, de ambos os sexos, com aptidão física e mental para participar da pesquisa e que assinaram o Termo de Consentimento Livre e Esclarecido (TCLE).

Os critérios de exclusão foram: menores de 18 anos, pacientes obesos que não apresentavam cardiopatias, pacientes cardiopatas não obesos, pacientes com doença cardíaca congênita, grávidas e indivíduos que não aceitaram participar da pesquisa.

Foram utilizados dados do prontuário clínico dos pacientes internados quanto ao tipo de doença cardiovascular, presença de comorbidades e história familiar.

\subsection{Avaliação do perfil nutricional}

Foi aferido o peso atual em kilograma $(\mathrm{Kg})$ utilizando uma balança eletrônica calibrada e a estatura em metros $(m)$ foi aferida através de estadiômetro.

O índice de massa corporal (IMC) foi calculado a partir dos dados de peso atual e estatura, considerando-se para os indivíduos participantes classificados como obesos o IMC a partir de $30 \mathrm{Kg} / \mathrm{m}^{2}$ segundo a Organização Mundial da Saúde (1997) para adultos e acima de $27 \mathrm{Kg} / \mathrm{m}^{2}$ para idosos, de acordo Lipschitz (1994).

A circunferência da cintura em centímetros $(\mathrm{cm})$ foi verificada no ponto médio entre a última costela e a crista ilíaca. Os valores de circunferência da cintura associados ao desenvolvimento de fatores de risco relacionados a obesidade e doenças cardiovasculares são $\geq 102 \mathrm{~cm}$ para homens e $\geq 88 \mathrm{~cm}$ para mulheres (NATIONAL INSTITUTES OF HEALTH, 2001).

O consumo alimentar foi avaliado por meio da aplicação do questionário de frequência alimentar (QFA) adaptado de Figueira, Rocha \& Araújo (2010), com a seleção de alimentos categorizados como "de risco" e "protetores" para a síndrome 
metabólica e doenças cardiovasculares, com as frequências de consumo diária, semanal, mensal e raro ou nunca.

\subsection{Avaliação do perfil clínico e bioquímico}

A medida da pressão arterial foi realizada por profissional capacitado por meio de esfigmomanômetro de coluna de mercúrio com técnica auscultatória, devidamente calibrada, sendo o valor considerado a média de três medidas, conforme recomendações da VI Diretriz Brasileira de Hipertensão Arterial de 2010 (BRASIL, 2010). Os exames bioquímicos (glicemia de jejum, triglicerídeos séricos e colesterol HDL sérico) foram realizados pelo Laboratório de Bioquímica do Hospital Universitário (HUMAP-EBSERH), de acordo com prescrição médica.

\subsection{Avaliação da Síndrome Metabólica}

Para a identificação da SM foram utilizados os critérios segundo o National Cholesterol Education Program's Adult Treatment Panel III (NCEP-ATP III, 2002), que preconiza que o indivíduo deve possuir pelo menos três dos componentes descritos no Quadro 1 (NATIONAL INSTITUTES OF HEALTH, 2001).

Quadro 1. Fatores de risco para Síndrome Metabólica.

\begin{tabular}{|lc|}
\hline Fatores de Risco & Critérios $^{\star}$ \\
\hline Circunferência da cintura & \\
Homens & $\geq 102 \mathrm{~cm}$ \\
Mulheres & $\geq 88 \mathrm{~cm}$ \\
Triglicerídeos & $\geq 150 \mathrm{mg} / \mathrm{dL}$ \\
HDL-colesterol** & \\
Homens & $<40 \mathrm{mg} / \mathrm{dL}$ \\
Mulheres & $<50 \mathrm{mg} / \mathrm{dL}$ \\
Pressão arterial & $\geq 130 \mathrm{mmHg} \mathrm{e} 85 \mathrm{mmHg}$ \\
Glicemia de jejum & $\geq 110 \mathrm{mg} / \mathrm{DI}$ \\
\hline
\end{tabular}

${ }^{\star}$ Segundo critérios do NCEP-ATP III; ${ }^{* *} \mathrm{HDL}-$ colesterol (high density lipoprotein-colesterol).

\subsection{Análise Estatística}

Os dados foram organizados e analisados através software SPSS (Statistical Package for the Social Sciences) versão 18.0. Foi realizada uma análise descritiva dos resultados por meio das frequências absolutas e relativas para as variáveis categóricas e da média \pm desvio padrão (DP) para as variáveis numéricas.

Para a comparação das variáveis categóricas foi utilizado o teste qui-quadrado e para avaliação das variáveis numéricas foi utilizado o Teste $t$ de Student. Foram considerados significativos os resultados com $\mathrm{p}<0,05$.

\section{RESULTADOS}

\subsection{Caracterização da população}

Foram avaliados 33 indivíduos que atendiam aos critérios de inclusão da presente pesquisa, sendo $51,52 \%$ homens e $48,48 \%$ mulheres. Ao avaliar os indivíduos por faixa etária, verificou-se que $42,42 \%$ eram adultos (20 a 59 anos) e $57,58 \%$ eram idosos (acima de 60 anos).

A média de idade de todos os participantes foi de 60,94 $\pm 1,65$ anos. Avaliandose a idade por sexo, não se encontrou diferença significativa (homens: 59,31 $\pm 9,25$ anos; mulheres: $62,47 \pm 9,79$ anos, $p=0,348)$. 
A Tabela 1 apresenta o tipo de doença cardiovascular, presença de comorbidades e história familiar de obesos cardiopatas, de acordo com sexo e idade. Destacando-se a cardiopatia mais prevalente entre os grupos o Infarto Agudo do Miocárdio $(30,3 \%)$ e a hipertensão arterial foi a mais prevalente co-morbidade.

Tabela 1. Caracterização de obesos cardiopatas, de acordo com sexo e idade, atendidos em um hospital universitário de Campo Grande, MS.

\begin{tabular}{|c|c|c|c|c|c|c|c|c|c|c|}
\hline \multirow{2}{*}{ Variáveis } & \multicolumn{2}{|c|}{$\begin{array}{c}\text { Total } \\
(\mathrm{n}=33)\end{array}$} & \multicolumn{2}{|c|}{$\begin{array}{c}\text { Homens } \\
(\mathrm{n}=16)\end{array}$} & \multicolumn{2}{|c|}{$\begin{array}{c}\text { Mulheres } \\
(\mathrm{n}=17)\end{array}$} & \multicolumn{2}{|c|}{$\begin{array}{c}\text { Adultos } \\
(\mathrm{n}=14)\end{array}$} & \multicolumn{2}{|c|}{$\begin{array}{l}\text { Idosos } \\
(\mathrm{n}=19)\end{array}$} \\
\hline & $\mathbf{n}$ & $\%$ & $\mathbf{N}$ & $\%$ & $\mathbf{n}$ & $\%$ & $\mathbf{n}$ & $\%$ & $\mathbf{n}$ & $\%$ \\
\hline \multicolumn{11}{|l|}{ Diagnóstico principal } \\
\hline DAC* Obstrutiva & 3 & 9,09 & 2 & 12,5 & 1 & 3,03 & - & - & 3 & 15,79 \\
\hline Fibrilação atrial & 2 & 6,06 & 1 & 6,25 & 1 & 5,88 & 1 & 7,14 & 1 & 5,26 \\
\hline $\mid A M^{* *}$ & 10 & 30,3 & 3 & 18,75 & 7 & 41,17 & 2 & 14,29 & 3 & 15,79 \\
\hline Insuficiência Cardíaca & 5 & 15,15 & 2 & 12,5 & 3 & 17,64 & 4 & 28,57 & 1 & 5,26 \\
\hline Outras ${ }^{\star * *}$ & 13 & 39,39 & 8 & 50 & 5 & 29,41 & 7 & 50 & 11 & 57,9 \\
\hline \multicolumn{11}{|l|}{ Co-morbidades } \\
\hline DAC & 2 & 6,06 & 2 & 12,5 & - & - & - & - & 2 & 10,52 \\
\hline Diabetes Mellitus (DM) & 1 & 3,03 & & - & 1 & 5,88 & - & - & 1 & 5,26 \\
\hline Hipertensão & 12 & 36,36 & 7 & 43,75 & 5 & 29,41 & 8 & 57,14 & 4 & 21,05 \\
\hline Hipertensão e DAC & 2 & 6,06 & 1 & 6,25 & 1 & 5,88 & 1 & 7,14 & 1 & 5,26 \\
\hline Hipertensão e DM & 11 & 33,33 & 5 & 31,25 & 6 & 35,29 & 2 & 14,28 & 9 & 47,36 \\
\hline Hipertensão, DM e DAC & 4 & 12,12 & 1 & 6,25 & 3 & 17,65 & 2 & 14,28 & 2 & 10,52 \\
\hline \multirow{2}{*}{\multicolumn{11}{|c|}{ História familiar }} \\
\hline & & & & & & & & & & \\
\hline DM & 1 & 3,03 & 1 & 6,25 & - & - & 1 & 5,26 & - & - \\
\hline Doença cardiovascular (DC) & 1 & 3,03 & 1 & 6,25 & - & - & - & - & 1 & 7,14 \\
\hline Hipertensão & 6 & 18,18 & 1 & 6,25 & 5 & 29,41 & 1 & 5,26 & 5 & 35,71 \\
\hline Hipertensão e DM & 3 & 9,09 & 1 & 6,25 & 2 & 11,77 & 1 & 5,26 & 2 & 14,28 \\
\hline Hipertensão e DC & 4 & 30,3 & 2 & 12,5 & 2 & 11,77 & 1 & 5,26 & 3 & 21,42 \\
\hline Hipertensão e obesidade & 2 & 6,06 & - & - & 2 & 11,77 & 1 & 5,26 & 1 & 7,14 \\
\hline Hipertensão, DM e DC & 3 & 9,09 & 2 & 12,5 & 1 & 5,87 & 2 & 10,52 & 1 & 7,14 \\
\hline Hipertensão, DM e obesidade & 3 & 9,09 & 1 & 6,25 & 2 & 11,77 & 1 & 5,26 & 2 & 14,28 \\
\hline $\begin{array}{l}\text { Hipertensão, DM, DC e } \\
\text { obesidade }\end{array}$ & 1 & 3,03 & 1 & 6,25 & - & - & - & - & 1 & 7,14 \\
\hline Obesidade & 2 & 6,06 & 1 & 6,25 & 1 & 5,87 & 2 & 10,52 & - & - \\
\hline Nenhuma & 7 & 21,21 & 5 & 31,25 & 2 & 11,77 & 4 & 21,05 & 3 & 21,42 \\
\hline
\end{tabular}

${ }^{*}$ DAC = Doença Arterial Coronariana; ${ }^{* *} I A M=$ Infarto Agudo do Miocárdio; ${ }^{* * *}$ Outras: Angina pectoris, fibrilaçã22o atrial aguda invertida, Acidente Vascular Cerebral, Insuficiência Cardíaca, Bloqueio Atrioventricular Total, Comunicação Interatrial, Endocardite Infecciosa, Estenose aórtica, Taquicardíaca ventricular, Dispneia, Síndrome Coronariana Aguda, Implante de Prótese Aórtica, Intervenção Coronariana Multiarterial, Isquemina miocárdica, Plastia mitral prévia, Pós-Operatório de Transposição Total de Troncos Supra Aórtica.

\subsection{Avaliação do perfil nutricional}

A Tabela 2 apresenta o perfil antropométrico de obesos cardiopatas, de acordo com sexo e idade. Devido a obesidade estar relacionada a um fator de inclusão do presente estudo, verificou-se para todos os grupos de comparação que os valores de IMC estavam acima de $30 \mathrm{Kg} / \mathrm{m}^{2}$, independente do sexo ou idade. Além disso, a média da circunferência da cintura apresentou-se elevada em todos os grupos de participantes (homens $105,59 \mathrm{~cm}$ vs mulheres $101,88 \mathrm{~cm}$, com $\mathrm{p}=0,156$; adultos $102,51 \mathrm{~cm}$ vs idosos $104,53 \mathrm{~cm}$, com $\mathrm{p}=0,451)$, caracterizando alto risco cardiovascular, porém sem apresentarem diferença significativa entre os grupos. 
Tabela 2. Perfil antropométrico de obesos cardiopatas, de acordo com sexo e idade, atendidos em um hospital universitário de Campo Grande, MS.

\begin{tabular}{|c|c|c|c|c|c|c|c|}
\hline Variáveis & $\begin{array}{c}\text { Total } \\
(n=33)\end{array}$ & $\begin{array}{c}\text { Homens } \\
(n=16)\end{array}$ & $\begin{array}{c}\text { Mulheres } \\
(n=17)\end{array}$ & $\begin{array}{c}p \\
\text { valor* }\end{array}$ & $\begin{array}{c}\text { Adultos } \\
(n=14)\end{array}$ & $\begin{array}{l}\text { Idosos } \\
(n=19)\end{array}$ & $\begin{array}{c}p \\
\text { valor* }\end{array}$ \\
\hline Peso (Kg) & $84,65 \pm 2,11$ & $92,38 \pm 11,11$ & $77,38 \pm 7,93$ & 0,0001 & $86,65 \pm 10,54$ & $83,18 \pm 13,27$ & 0,426 \\
\hline Altura (cm) & $1,60 \pm 0,01$ & $1,66 \pm 0,03$ & $1,54 \pm 0,05$ & 0,0001 & $1,61 \pm 0,07$ & $1,60 \pm 0,08$ & 0,761 \\
\hline $\operatorname{IMC}\left(\mathrm{Kg} / \mathrm{m}^{2}\right)^{\star *}$ & $32,7 \pm 0,53$ & $33,21 \pm 3,33$ & $32,22 \pm 2,81$ & 0,363 & $33,34 \pm 3,07$ & $32,23 \pm 3,05$ & 0,311 \\
\hline $\mathrm{CC}(\mathrm{cm})^{\star * *}$ & $103,67 \pm 7,46$ & $105,59 \pm 7,65$ & $101,88 \pm 7,02$ & 0,156 & $102,51 \pm 6,59$ & $104,53 \pm 8,10$ & 0,451 \\
\hline
\end{tabular}

A Tabela 3 apresenta os resultados da frequência de ingestão de alimentos "de risco" e "protetores" para doenças cardiovasculares de obesos cardiopatas, de acordo com sexo e idade.

Tabela 3. Frequências de ingestão de alimentos de risco e protetores para doenças cardiovasculares de obesos cardiopatas, segundo o sexo atendidos em um hospital universitário de Campo Grande, MS.

\begin{tabular}{|c|c|c|c|c|c|c|c|c|c|}
\hline \multirow{3}{*}{ Alimentos } & \multicolumn{4}{|c|}{ Homens $(n=16)$} & \multicolumn{4}{|c|}{ Mulheres $(n=17)$} & \multirow{3}{*}{$\begin{array}{c}\mathrm{p} \\
\text { valor* }\end{array}$} \\
\hline & \multicolumn{2}{|c|}{ Diária } & \multicolumn{2}{|c|}{$\begin{array}{l}\text { Raro ou } \\
\text { nunca }\end{array}$} & \multicolumn{2}{|c|}{ Diária } & \multicolumn{2}{|c|}{$\begin{array}{c}\text { Raro ou } \\
\text { nunca }\end{array}$} & \\
\hline & $\mathrm{n}$ & $\%$ & $\mathrm{~N}$ & $\%$ & $\mathrm{~N}$ & $\%$ & $\mathrm{n}$ & $\%$ & \\
\hline ALIMENTOS “DE RISCO” & & & & & & & & & \\
\hline Carnes & 14 & 87,5 & 1 & 6,3 & 11 & 64,7 & 1 & 5,9 & 1,000 \\
\hline Embutidos e enlatados & - & - & 7 & 43,8 & - & - & 11 & 64,7 & - \\
\hline Temperos prontos & 4 & 25,0 & 11 & 68,8 & 3 & 17,7 & 13 & 76,5 & 0,685 \\
\hline Ovos & 1 & 6,3 & 4 & 25,0 & 2 & 11,8 & 8 & 47,1 & 1,000 \\
\hline Açúcar & 13 & 81,3 & 1 & 6,3 & 9 & 52,9 & 7 & 41,2 & 0,039 \\
\hline Manteiga/margarina & 11 & 68,8 & 2 & 12,5 & 8 & 47,1 & 6 & 35,3 & 0,209 \\
\hline Leite integral & 5 & 31,3 & 6 & 37,5 & 8 & 47,1 & 2 & 11,8 & 0,183 \\
\hline Queijos amarelos & 2 & 12,5 & 4 & 25,0 & 2 & 11,8 & 8 & 47,1 & 0,604 \\
\hline Doces & 1 & 6,3 & 10 & 62,5 & 1 & 5,9 & 14 & 82,4 & 1,000 \\
\hline Frituras & 8 & 50,0 & 3 & 18,8 & 5 & 29,4 & 4 & 23,5 & 0,642 \\
\hline Refrigerante & 2 & 12,5 & 6 & 37,5 & - & - & 11 & 64,7 & 0,267 \\
\hline Suco industrializado & 2 & 12,5 & 9 & 56,3 & 3 & 17,7 & 13 & 76,5 & 1,000 \\
\hline Bebidas alcoólicas & 2 & 12,5 & 14 & 87,5 & - & - & 16 & 94,1 & 0,484 \\
\hline Pães e bolos & 12 & 75,0 & - & - & 11 & 64,7 & 4 & 23,5 & 0,106 \\
\hline $\begin{array}{l}\text { Biscoito recheado } \\
\text { Al IMFNTOS "PROTFTORFS" }\end{array}$ & - & - & 15 & 93,8 & - & - & 15 & 88,2 & - \\
\hline $\begin{array}{l}\text { ALIMENTOS "PROTETORES" } \\
\text { Carne de peixe }\end{array}$ & 1 & 6,3 & 5 & 31,3 & - & - & 6 & 35,3 & 1,000 \\
\hline Leite desnatado & - & - & 15 & 93,8 & 1 & 5,9 & 16 & 94,1 & 1,000 \\
\hline Queijo branco & - & - & 13 & 81,3 & - & - & 17 & 100,0 & - \\
\hline Legumes & 7 & 43,8 & - & - & 11 & 64,7 & 1 & 5,9 & 1,000 \\
\hline Verduras & 11 & 68,8 & - & - & 12 & 70,6 & 1 & 5,9 & 1,000 \\
\hline Feijão & 10 & 62,5 & 4 & 25,0 & 11 & 64,7 & 2 & 11,8 & 0,648 \\
\hline Soja & 1 & 6,3 & 15 & 93,8 & - & - & 16 & 94,1 & 1,000 \\
\hline Suco de fruta & 1 & 6,3 & 3 & 18,8 & 8 & 47,1 & - & - & 0,018 \\
\hline Frutas & 6 & 37,5 & - & - & 12 & 70,6 & 1 & 5,9 & 1,000 \\
\hline Azeite de olive & 4 & 25,0 & 9 & 56,3 & 5 & 29,4 & 9 & 52,9 & 1,000 \\
\hline Cereais integrais & - & - & 15 & 93,8 & 2 & 11,8 & 12 & 70,6 & 0,224 \\
\hline
\end{tabular}

* Valor de p obtido através do Teste Exato de Fisher na comparação por sexo.

Foi observado que mais de $50 \%$ dos homens consomem diariamente alimentos "de risco", com destaque para carnes, açúcar, manteiga/margarina, queijos amarelos, frituras e pães e bolos, e que corresponde a $40 \%$ de todos os alimentos de risco. Quanto aos alimentos "protetores", mais de $50 \%$ dos homens e mulheres apresentam consumo raro ou nunca de leite desnatado, queijo branco, azeite de oliva e cereais integrais. Além disso, notou-se que o consumo diário de suco de fruta pelo grupo de 
mulheres apresentou-se 8 vezes maior quando comparado ao grupo de homens, com diferença significativa, e o mesmo ocorreu com o consumo de açúcar, onde mulheres apresentaram consumo significativamente maior em relação aos homens (Tabela 3).

Entre relação a adultos e idosos não foi encontrada diferença significativa, apesar de idosos consumirem o dobro de feijão e frutas em relação aos adultos, os quais correspondem a apenas $18,2 \%$ de todos os alimentos protetores.

Desta forma, é observado um baixo consumo de alimentos "protetores" pelo grupo de homens $(23,3 \%)$ e adultos $(26,6 \%)$, em relação as mulheres $(33,2 \%)$ e idosos $(29,68 \%)$, respectivamente.

Tabela 4. Frequências de ingestão de alimentos de risco e protetores para doenças cardiovasculares de obesos cardiopatas, segundo a idade atendidos em hospital universitário de Campo Grande, MS.

\begin{tabular}{|c|c|c|c|c|c|c|c|c|c|}
\hline \multirow{3}{*}{ Alimentos } & \multicolumn{4}{|c|}{ Adultos $(n=14)$} & \multicolumn{4}{|c|}{ Idosos $(n=19)$} & \multirow{3}{*}{$\begin{array}{c}p \\
\text { valor* }\end{array}$} \\
\hline & \multicolumn{2}{|c|}{ Diária } & \multicolumn{2}{|c|}{$\begin{array}{c}\text { Raro ou } \\
\text { nunca }\end{array}$} & \multicolumn{2}{|c|}{ Diária } & \multicolumn{2}{|c|}{$\begin{array}{l}\text { Raro ou } \\
\text { nunca }\end{array}$} & \\
\hline & $\mathrm{n}$ & $\%$ & $\mathrm{~N}$ & $\%$ & $\mathrm{n}$ & $\%$ & $\mathrm{n}$ & $\%$ & \\
\hline ALIMENTOS “DE RISCO” & & & & & & & & & \\
\hline Carnes & 11 & 78,6 & - & - & 14 & 73,7 & 2 & 10,5 & 0,499 \\
\hline Embutidos e enlatados & - & - & 8 & 57,1 & 5 & 26,3 & 10 & 52,6 & - \\
\hline Temperos prontos & 3 & 21,4 & 10 & 71,4 & 4 & 21,1 & 14 & 73,7 & 1 \\
\hline Ovos & 2 & 14,3 & 4 & 28,6 & 1 & 5,3 & 8 & 42,1 & 0,525 \\
\hline Açúcar & 11 & 78,6 & 1 & 7,1 & 11 & 57,9 & 7 & 36,8 & 0,099 \\
\hline Manteiga/margarina & 10 & 71,4 & 4 & 28,6 & 9 & 47,4 & 4 & 21,1 & 1 \\
\hline Leite integral & 8 & 57,1 & 3 & 21,4 & 5 & 26,3 & 5 & 26,3 & 0,387 \\
\hline Queijos amarelos & 2 & 14,3 & 4 & 58,6 & 2 & 10,5 & 8 & 42,1 & 0,604 \\
\hline Doces & 1 & 7,1 & 11 & 78,6 & 1 & 5,3 & 14 & 73,7 & 1 \\
\hline Frituras & 7 & 50,0 & 2 & 14,3 & 6 & 31,6 & 5 & 26,3 & 0,374 \\
\hline Refrigerante & 2 & 14,3 & 7 & 50,0 & 1 & 5,3 & 10 & 52,6 & 0,483 \\
\hline Suco industrializado & 3 & 21,4 & 10 & 71,4 & 1 & 5,3 & 12 & 63,2 & 0,593 \\
\hline Bebidas alcoólicas & 1 & 7,1 & 13 & 92,9 & 1 & 5,3 & 17 & 89,5 & 1 \\
\hline Pães e bolos & 11 & 78,6 & 1 & 7,1 & 12 & 63,2 & 3 & 15,8 & 0,605 \\
\hline $\begin{array}{l}\text { Biscoito recheado } \\
\text { ALIMENTOS "PROTETORES" }\end{array}$ & - & - & 12 & 85,7 & - & - & 17 & 89,5 & - \\
\hline Carne de peixe & - & - & 2 & 14,3 & 1 & 5,3 & 9 & 47,4 & 1 \\
\hline Leite desnatado & - & - & 13 & 92,9 & 1 & 5,3 & 18 & 94,7 & 1 \\
\hline Queijo branco & - & - & 12 & 85,7 & - & & 18 & 94,7 & - \\
\hline Legumes & 9 & 64,3 & - & - & 9 & 47,4 & 1 & 5,3 & 1 \\
\hline Verduras & 10 & 71,4 & - & - & 13 & 68,4 & 1 & 5,3 & 1 \\
\hline Feijão & 7 & 50,0 & 3 & 21,4 & 14 & 73,7 & 3 & 15,8 & 0,638 \\
\hline Soja & - & - & 14 & 100,0 & 1 & 5,3 & 17 & 89,5 & 1 \\
\hline Suco de fruta & 4 & 28,6 & - & - & 5 & 26,3 & 3 & 15,8 & 0,491 \\
\hline Frutas & 6 & 42,9 & 1 & 7,1 & 12 & 63,2 & - & - & 0,368 \\
\hline Azeite de olive & 4 & 28,6 & 8 & 57,1 & 5 & 26,3 & 10 & 52,6 & 1 \\
\hline Cereais integrais & 1 & 7,1 & 13 & 92,9 & 1 & 5,3 & 14 & 73,7 & 1 \\
\hline
\end{tabular}

* Valor de p obtido através do Teste Exato de Fisher na comparação por sexo.

\subsection{Avaliação do perfil clínico e bioquímico}

A Tabela 5 demonstra o perfil clínico e bioquímico de obesos cardiopatas, de acordo com sexo e idade. Para a maioria dos grupos não foi encontrada diferença, porém todos apresentaram níveis elevados de glicemia de jejum e triglicerídeos e baixos níveis de HDL-c comparado com recomendações. Todos os participantes estavam normotensos, provavelmente devido à medicação anti-hipertensiva 
Tabela 5. Perfil clínico e bioquímico de obesos cardiopatas, de acordo com sexo e idade, atendidos em um hospital universitário de Campo Grande, MS.

\begin{tabular}{lccccccc}
\hline Variáveis & $\begin{array}{c}\text { Total } \\
(\mathbf{n}=\mathbf{3 3})\end{array}$ & $\begin{array}{c}\text { Homens } \\
(\mathbf{n}=\mathbf{1 6})\end{array}$ & $\begin{array}{c}\text { Mulheres } \\
(\mathbf{n}=\mathbf{1 7})\end{array}$ & $\begin{array}{c}\boldsymbol{p} \\
\text { valor }\end{array}$ & $\begin{array}{c}\text { Adultos } \\
(\mathbf{n}=\mathbf{1 4})\end{array}$ & $\begin{array}{c}\text { Idosos } \\
(\mathbf{n}=19)\end{array}$ & $\begin{array}{c}\boldsymbol{p} \\
\text { valor }\end{array}$ \\
\hline Pressão sistólica & $126,21 \pm$ & $125,06 \pm$ & $127,29 \pm$ & 0,755 & $126,42 \pm$ & $126,05 \pm$ & 0,959 \\
(mmHg) & 3,49 & 18,80 & 21,70 & & 18,49 & 21,64 & \\
Pressão diastólica & $81,15 \pm$ & $84,37 \pm$ & $78,11 \pm$ & 0,490 & $84,64 \pm$ & $78,57 \pm$ & 0,508 \\
(mmHg) & 4,44 & 34,88 & 11,86 & & 18,80 & 29,75 & \\
Glicose de jejum & $157,66 \pm$ & $163,56 \pm$ & $152,11 \pm$ & 0,706 & $134,07 \pm$ & $175,05 \pm$ & 0,176 \\
(mg/dL) & 14,82 & 72,56 & 97,46 & & 64,19 & 95,72 & \\
Triglicerídeos & $189,36 \pm$ & $214,37 \pm$ & $165,82 \pm$ & 0,208 & $212,14 \pm$ & $172,57 \pm$ & 0,313 \\
(mg/dL) & 19,07 & 141,45 & 63,52 & & 150,36 & 65,64 & \\
HDL-c (mg/dL) & $36,98 \pm$ & $31,92 \pm$ & $41,74 \pm$ & $0,008^{*}$ & $36,32 \pm$ & $37,47 \pm$ & 0,772 \\
& 1,92 & 11,43 & 8,45 & & 9,74 & 12,14 & \\
\hline
\end{tabular}

Valores expressos em média e desvio padrão (DP). ${ }^{*}$ Valor de p obtido através do Teste $t$-student na comparação por sexo e idade. ${ }^{*} \mathrm{HDL}-\mathrm{c}$ (high density lipoprotein-colesterol).

\subsection{Avaliação da Síndrome Metabólica}

$\mathrm{Na}$ Figura 1 é apresentada a distribuição do percentual dos componentes da síndrome metabólica entre obesos cardiopatas de acordo com sexo e idade. De acordo com os critérios do NCEP-ATP III, a prevalência de Síndrome Metabólica foi de $84,85 \%$ do total de obesos cardiopatas $(n=33)$. Destaca-se que entre os adultos essa prevalência foi de $85,71 \%$ e entre os idosos foi de $100 \%$.

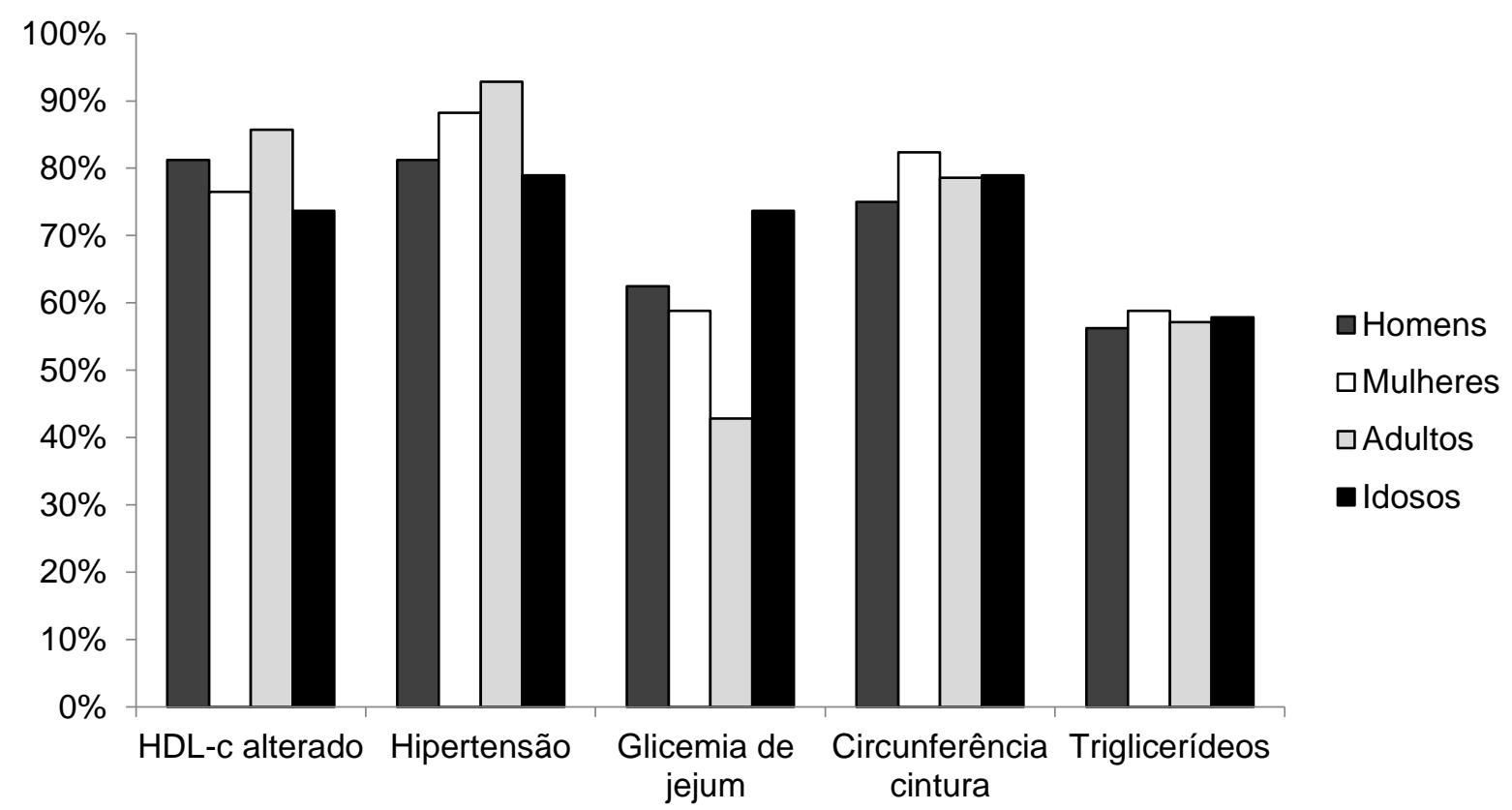

Figura 1. Distribuição percentual dos componentes da síndrome metabólica (SM) entre obesos cardiopatas de acordo com sexo e idade, atendidos em um hospital universitário de Campo Grande, MS.

\section{DISCUSSÃO}

Nas últimas décadas as Doenças Cardiovasculares (DCV) foram responsáveis por $30 \%$ da mortalidade, ou seja, 17 milhões de pessoas no mundo(SANTOS et al., 2013). Especificamente o infarto agudo do miocárdio apresenta-se como principal causa isolada de morte no Brasil. Estima-se que dois terços das mortes súbitas por doenças cardíacas ocorrem fora do ambiente hospitalar e apenas $20 \%$ das pessoas 
com relato de dor torácica aguda chegam à emergência antes de duas horas do início desses sinais, sendo o rápido atendimento desses pacientes, após o início dos primeiros sintomas, um importante fator que contribui para a redução desta mortalidade (BASTOS, 2012).

Vale ressaltar que o histórico familiar de doenças representa no indivíduo um fator de risco não modificável que também contribui para a constatação de um determinado diagnóstico incluindo as DCV. Além disso, reflete as consequências da susceptibilidade genética que interage com o ambiente e o estilo de vida (TAVARES, 2008).

No presente estudo a hipertensão arterial apresentou-se como a principal comorbidade, sendo também a mais referida no histórico familiar. Além disso, é o principal fator de risco para doença coronariana e outras doenças do aparelho circulatório, assim como compõe a síndrome metabólica (BRASIL, 2010). A hipertensão arterial é considerada um relevante problema de saúde pública e sua prevalência na população brasileira é superior a $60 \%$ na faixa etária acima de 65 anos (BRASIL, 2010).

Além disso, a elevada incidência de IAM no presente estudo, principalmente entre mulheres, apresentou-se semelhante a estudo realizado em Salvador, o qual, ao analisar o gênero e a letalidade hospitalar das síndromes coronarianas agudas, comprovou-se que o risco de morte em mulheres foi duas vezes maior que em homens (PASSOS, 2000).

Ao analisar o perfil antropométrico, foi observada uma diferença significativa entre homens e mulheres com relação ao peso e altura no presente estudo. Entretanto, já era prevista e decorrente da diferença existente entre os sexos (CARVALHO, PIRES, 1999). O declínio do peso entre os indivíduos idosos apresentase associado ao envelhecimento, sendo especialmente devido à diminuição na massa magra e a diminuição da densidade óssea (MATSUDO, MATSUDO, BARROS, 2000).

Devido a obesidade ser um fator de inclusão do estudo, todos os indivíduos apresentaram IMC maior ou igual a $30 \mathrm{Kg} / \mathrm{m}^{2}$, porém identificaram-se dados de circunferência da cintura elevada em $100 \%$ dos participantes, onde foi observado que a elevação foi diretamente proporcional à idade. Resultado também esperado, pois este padrão de distribuição de gordura se altera com o envelhecimento, havendo um acréscimo de $40 \%$ da gordura intra-abdominal a partir da quinta década da vida, sendo que no sexo masculino a gordura subcutânea diminui na periferia, mas aumenta centralmente (tronco) e internamente (vísceras), e no sexo feminino a gordura subcutânea pode permanecer estável até os 45 anos, com aumento da circunferência da cintura e do quadril a partir dessa idade (MATSUDO, MATSUDO, BARROS, 2000).

A relevância dos fatores de risco ambientais e hereditários, quando relacionada ao aumento de chances para o desenvolvimento de DCNT, acarretam considerável impacto na qualidade de vida do indivíduo e o surgimento de doenças (BRASIL, 2013).

Nesse contexto, o parâmetro dietético é um importante fator ambiental que exerce também grande influência na escolha de determinados hábitos alimentares (BRASIL, 2014; ENES, SLATER, 2010). Relata-se que óleos, gorduras, sal e açúcar são produtos cujo consumo excessivo pode ser prejudicial à saúde. A ingestão de sódio e gorduras saturadas aumenta 0 risco de doenças cardíacas, enquanto o consumo excessivo de açúcar aumenta o risco de obesidade e várias outras doenças crônicas, colocando a população em uma situação de risco (BRASIL, 2013).

No presente estudo, foi possível avaliar o elevado consumo de alimentos considerados "de risco" para a Síndrome Metabólica, como: carnes, açúcar, 
manteiga/margarina e frituras, quando comparados ao consumo de alimentos considerados "protetores" como as frutas, verduras, legumes e feijão.

A relação entre o elevado consumo de gordura como fator de risco cardiovascular pode ser considerado como condição que contribui para o atual estado dos indivíduos cardiopatas avaliados (LOTTENBERG, 2009). Foi possível identificar que as mulheres apresentaram maior tendência no consumo de frutas, legumes e verduras em relação aos homens, que corresponde a $27,3 \%$ de todos os alimentos protetores pesquisados, porém sem diferença significativa entre os sexos. O mesmo foi observado no estudo realizado sobre os fatores associados ao consumo de frutas, legumes e verduras em adultos da cidade de São Paulo, o qual constatou uma maior frequência de consumo de frutas, legumes e verduras entre as mulheres (FIGUEIREDO, JAIME, MONTEIRO, 2008).

Mediante os resultados do Questionário de Frequência Alimentar, foi possível verificar entre homens e mulheres o mesmo padrão de consumo de alimentos de "risco", os quais se destacaram o consumo diário de: carnes, açúcar, manteiga/margarina, queijos amarelos, frituras e pães e bolos, durante todos os dias da semana. Entre adultos, destacou-se o diário consumo de: carnes, açúcar, leite integral, frituras, manteiga/margarina e pães e bolos, porém entre os idosos, verificouse o consumo com frequência diária somente de: carnes, açúcar, pães e bolos. Ao se comparar o consumo de adultos e idosos, foi possível verificar grande semelhança e um padrão alimentar com predominância de alimentos categorizados de risco.

De um modo geral, foi notável a existência de um frequente consumo de gorduras e açúcares na alimentação dos participantes da pesquisa. Vale ressaltar que a ingestão de refeições ricas nesses macronutrientes constitui um motivo para precaução, pois o excesso de gordura eleva a densidade energética da dieta, predispondo assim ao surgimento da obesidade e de outras doenças associadas (BRASIL, 2014b).

No presente estudo, os alimentos considerados protetores para SM para homens e mulheres foram o leite desnatado, queijo branco, azeite de oliva e cereais integrais, apresentando grande semelhança entre os sexos, sem significativa diferença de consumo. Entre adultos, observou-se alta prevalência de frequência de consumo "raro" ou "nunca" para legumes e soja. Já entre os idosos, destacou-se o consumo "raro" ou "nunca" para queijo branco, soja, azeite de oliva e cereais integrais. Os alimentos protetores citados anteriormente também se encontram presentes na dieta mediterrânea e são considerados de grande importância na redução do risco de doenças cardiovasculares (BRASIL, 2013b).

Pinho et al. (2012) realizou a correlação entre variáveis nutricionais e clínicas de idosos cardiopatas, avaliou também o consumo alimentar destes indivíduos, mediante a aplicação de um questionário de frequência alimentar. Foi verificado um significativo consumo de açúcares e doces, óleos e gorduras, feijão e sementes, cereais, carnes e ovos. Os resultados do estudo supracitado assemelham-se ao presente trabalho pela alta ingestão de grande parte destes alimentos, exceto 0 consumo de sementes, cereais e doces.

No perfil clínico dos pacientes cardiopatas, a pressão arterial apresentou-se normalizada e sem diferença significativa entre os grupos devido ao uso de antihipertensivo durante a internação, demonstrando um controle efetivo com 0 tratamento medicamentoso, diferente do constatado no estudo realizado no Estado do Rio Grande do Sul, que avaliou a prevalência, reconhecimento e controle da hipertensão arterial sistêmica. Neste estudo, foi observado um elevado número de indivíduos que seguiam o tratamento, mas não apresentavam controle adequado, 
representando um grande desafio a ser enfrentado pelo sistema de saúde (GUS et al., 2004). Entretanto, esse fator de risco para SM, mesmo controlado, foi incluído a fim de justificar a doença cardiovascular atual.

Um estudo realizado em Portugal avaliou a prevalência e controle da hipertensão, demonstrando também que a diferença dos valores da pressão arterial sistólica e diastólica entre o sexo masculino e feminino tende a atenuar com a idade, sendo esse fator também associado a alterações metabólicas, com consequente aumento do risco de eventos cardiovasculares fatais e não fatais (MATSUDO, MATSUDO, BARROS, 2000; BRASIL, 2010).

Os índices de glicemia apresentados pelos avaliados mostraram-se elevados em todos os grupos decorrentes de um inadequado controle medicamentoso ou da influência da dieta, quando próximo ao período de aferição, durante a internação, sendo diferente dos resultados encontrados em estudo realizado com pacientes diabéticos (SILVA et al., 1999), onde foi verificado um controle adequado da glicemia em $50 \%$ dos pacientes analisados.

Os níveis de triglicérides encontraram-se elevados quando comparados com o padrão de referência superior ou igual a $150 \mathrm{mg} / \mathrm{dL}$ em todos os grupos, destacandose que entre homens e adultos foi verificado uma tendência de maiores níveis (NATIONAL INSTITUTES OF HEALTH, 2001). Estes resultados mostraram-se diferente de estudo realizado com 316 indivíduos obesos, atendidos no ambulatório de um hospital universitário em Salvador, onde foi encontrada uma reduzida frequência de hipertrigliceridemia, contudo grande parte destes pacientes possuíam outros fatores para caracterizar a síndrome metabólica (PORTO et al., 2002).

Foram encontrados reduzidos níveis de HDL-colesterol em todos os grupos sem diferença significativa. Com base em um estudo realizado em Bauru (BERTOLINO et al., 2006), onde foi analisada a influência do consumo alimentar no perfil de lipídios séricos em nipo-brasileiros, foi possível demonstrar que uma significativa redução no consumo total de gordura estava relacionada com a redução dos níveis séricos de LDL-colesterol e aumento do HDL-colesterol. Deste modo, a inadequada ingestão alimentar pode ser um fator de contribuição para a ocorrência da SM aliada às doenças cardiovasculares atuais.

Evidências epidemiológicas demonstram cada vez mais a associação entre síndrome metabólica e presença de alterações cardiovasculares, uma vez que, de forma independente, os fatores de risco que estão relacionados às DCV são os mesmos que compõem a síndrome. A coexistência desses fatores de risco eleva as taxas de eventos cardiovasculares e leva ao mau prognóstico do paciente, aumentando a mortalidade geral em cerca de 1,5 vezes e a cardiovascular em cerca de 2,5 vezes (QUIRINO et al., 2014). No presente estudo, encontrou-se alta prevalência de síndrome metabólica nos obesos cardiopatas estudados, sendo que, entre idosos, a identificação foi em $100 \%$ dos casos, logo pode ser considerado fator determinante para o atual diagnóstico dos pacientes.

Estudo realizado na América Latina demonstrou resultado semelhante ao avaliar a prevalência de SM, constatando índices ligeiramente maiores entre as mulheres em relação aos homens e a faixa etária com maior prevalência foi entre indivíduos acima de 50 anos de idade (MÁRQUEZ et al., 2011).

\section{CONSIDERAÇÕES FINAIS}

Foi identificada uma alta prevalência de síndrome metabólica nos grupos estudados, com destaque para mulheres e idosos. Evidenciou-se possível influência 
dos fatores hereditários identificados através da história familiar, onde é marcante a presença de doenças crônicas relacionadas às doenças cardiovasculares. Com relação ao perfil antropométrico e bioquímico, foi observada circunferência da cintura aumentada em todos os grupos, podendo caracterizar acúmulo de gordura abdominal, associada altos níveis de glicemia, triglicérides e baixos níveis de HDL-C.

Com relação ao consumo alimentar notou-se um elevado consumo de alimentos "protetores" pelo grupo de mulheres e de idosos, e um elevado consumo de alimentos de risco entre homens e adultos. O padrão dietético encontrado no estudo, juntamente com o perfil clínico e antropométrico, pode justificar a alta prevalência de síndrome metabólica identificada nos pacientes obesos cardiopatas hospitalizados estudados. Diante disso, a presença de um nutricionista na equipe de saúde mostrase fundamental para uma adequada orientação alimentar, buscando primeiramente a prevenção da doença, o que possivelmente minimizará futuros agravos, além de proporcionar tratamento adequado e melhor qualidade de vida aos pacientes diagnosticados com a síndrome.

\section{REFERENCIAS}

BALLESTRERI, E.; MARCON, I.F.; TAVARES, R.G. Comparação de modelos de indução da síndrome metabólica: dieta com excesso de frutosa e dieta hiperlipidêmica. RBONE (Revista Brasileira de Obesidade, Nutrição e Emagrecimento), v. 9, n. 51, p. 96-103, 2015.

BASTOS, A.S. Tempo de chegada do paciente com infarto agudo do miocárdio em unidade de emergência. Rev. Bras. Cir. Cardiovasc. v. 27, n. 3, p. 411-8, 2012.

BERTOLINO, C.N.; CASTRO, T.G.; SARTORELLI, D.S.; FERREIRA, S.R.G; CARDOSO, M.A., et al. Influência do consumo alimentar de ácidos graxos trans no perfil de lipídios séricos em nipo-brasileiros de Bauru, São Paulo, Brasil. Cader. Saúde Públ. v. 22, n. 2, p. 357-64, 2006.

BRASIL. Diretriz de Doença Coronária Estável. Soc. Bras. Cardiol. 2014; 103 (Supl 2).

BRASIL. Guia Alimentar para a População Brasileira. 2ª Edição. Brasília: Ministério da Saúde; 2014.

BRASIL. I Diretriz Brasileira de Diagnóstico e Tratamento da Síndrome Metabólica. Arq. Bras. Cardiol. v. 84, 2005.

BRASIL. I Diretriz Brasileira de Prevenção Cardiovascular. Arquiv. Bras. Cardiol. v. 101, n. 6, 2013.

BRASIL. Portal da Inovação na Gestão do SUS [acesso em 27 nov 2015]. Disponível em: http://apsredes.org/site2013/vocesaudavel/2013/04/03/fatores-de-risco/

BRASIL. VI Diretriz Brasileira de Hipertensão. Arquiv. Bras. Cardiol. v. 95, n. 1, p. 1$51,2010$. 
CARVALHO, A.B.R.; PIRES NETO, C.S. Composição corporal através dos métodos da pesagem hidrostática e impedância bioelétrica em universitários. Rev. Bras. Cineantropom. Desempenho Hum. v. 1, n. 1, p. 18-23, 1999.

ENES, C.C.; SLATER, B. Obesidade na adolescência e seus principais fatores determinantes. Rev. Bras. Epidemiol. v. 13, n. 1, p. 163-71, 2010.

FIGUEIRA, M.S.; ROCHA, M.L.V; ARAÚJO, M.S. Avaliação nutricional de pacientes portadores de doenças cardiovasculares associadas à síndrome metabólica em Belém-PA. Rev Bras. Nutr. Clín. v. 25, n. 3, p. 224-32, 2010.

FIGUEIREDO, I.C.R.; JAIME, P.C.; MONTEIRO, C.A. Fatores associados ao consumo de frutas, legumes e verduras em adultos da cidade de São Paulo. Rev. Saúde Publ. v. 42, n. 5, p. 777-85, 2008.

GUS, I.; HARZHEIM, E.; ZASLAVSKY, C.; MEDINA, C.; GUS, M. Prevalência, Reconhecimento e Controle da Hipertensão Arterial Sistêmica no Estado do Rio Grande do Sul. Arquiv. Bras. Cardiol. v. 83, n. 5, p. 424-28, 2004.

INTERNATIONAL DIABETES FEDERATION. The IDF consensus worldwide de nition of the METABOLIC SYNDROME [acesso em 04 set 2014]. Disponível em: http://www.idf.org/webdata/docs/IDF Meta def final.pdf

LIPSCHITZ, D.A. Screening for nutritional status in the elderly. Prim. Care. v. 21, n. 1, p. $55-67,1994$.

LOTTENBERG, A.M.P. Importância da gordura alimentar na prevenção e no controle de distúrbios metabólicos e da doença cardiovascular. Arquiv. Bras. Endocrinol. e Metabol. v. 53, n. 5, p. 595-607, 2009.

MALTA, D.C.; SILVA, J.B. O Plano de Ações Estratégicas para o Enfrentamento das Doenças Crônicas Não Transmissíveis no Brasil e a definição das metas globais para o enfrentamento dessas doenças até 2025: uma revisão. Epidemiol. Serv. Saúde. v. 22, n. 1, p. 151-64, 2013.

MÁRQUEZ,F.S.; MACEDO,G.O.; VIRAMONTES,D.H.; FERNÁNDEZ,D.B.; SALAS,J. S.; VIZMANOS. B. The prevalence of metabolic syndrome in Latin America: a systematic review. Public Health Nutrition. v. 14, n. 10, p. 1702-13, 2011.

MATSUDO, S.M.; MATSUDO, V.K.R.; BARROS NETO, T.L. Impacto do envelhecimento nas variáveis antropométricas, neuromotoras e metabólicas da aptidão física. Rev. Bras. Cienc. Mov. v. 8, n. 4, p. 21-32, 2000.

NATIONAL INSTITUTES OF HEALTH . Detection, Evaluation, and Treatment of High Blood Cholesterol in Adults (Adult Treatment Panel III); 2001.

ORGANIZAÇÃO MUNDIAL DA SAÚDE. Classificação estatística internacional de doenças e problemas relacionados à saúde. São Paulo, SP: Editora da Universidade de São Paulo; 1997. 
PASSOS, L.C.S. Gênero e letalidade hospitalar das síndromes coronarianas agudas em Salvador-Bahia. Salvador: Universidade Federal da Bahia; 2000.

PINHO P.M.; SILVA, A.C.M; ARAÚJO, M.S.; REIS, C.P.; ALMEIDA, S.S.; BARROS, L.C.A.; et al. Correlação entre Variáveis Nutricionais e Clínicas de Idosos Cardiopatas. Rev. Bras. Cardiol. v. 25, n. 2, p. 132-42, 2012.

PORTO, M.C.V.; BRITO, C.I.; CALFA, A.D.F.; AMORAS, M.; VILLELA, N.B.; ARAÚJO, L.M.B. Perfil do Obeso Classe III do Ambulatório de Obesidade de Um Hospital Universitário de Salvador, Bahia. Arquiv. Bras. Endocrinol. e Metabol. v. 46, n. 6, p. 668-73, 2002.

QUIRINO, C.S.P.; MARANHÃO, R.V.A.; GIANNINI, D.T. Síndrome Metabólica em Pacientes Atendidos em Programa de Reabilitação Cardíaca. Rev. Bras. Cardiol. v. 27, n. 3, p. 180-88, 2014.

SANTOS, R.D.; GAGLIARDI, A.C.M.; XAVIER, H.T.; MAGNONI, C.D.; CASSANI, R.; LOTTENBERG, A.M.P.; et al. I Diretriz sobre o consumo de gorduras e saúde cardiovascular. Arq. Bras. Cardiol. v. 100, n. 1, p. 1-40, 2013.

SHILS, M.E.; SHIKE, M.; ROSS, A.C.; CABALLERO, B.; COUSINS, R.J. Nutrição moderna na saúde e na doença. 10ª edição. São Paulo: Manole; 2009.

SILVA JÚNIOR, G.R.; FUKS, A.G.; CUNHA, E.F.; CLEMENTE, E.F.S.; GOMES, M.B. Inter-relação de Variáveis Demográficas, Terapêutica Insulínica e Controle Glicêmico em Pacientes com Diabetes Mellitus do Tipo 1 Atendidos em Um Hospital Universitário. Arquiv. Bras. Endocrinol. e Metabol. v. 43, n. 2, p. 114-20, 1999.

TAVARES, P.A.P. História Familiar de Doença Coronária, Cuidados e Comportamentos em Saúde. Porto: U. PORTO; 2008.

TIRAPEGUI, J. Nutrição Fundamentos e Aspectos Atuais. 2a edição. São Paulo: Atheneu; 2006.

WORLD HEALTH ORGANIZATION. Global status report on noncommunicable diseases 2010 [acesso em 09 set 2014]. Disponível em: http://www.who.int/nmh/publications/ncd_report_full_en.pdf 